\title{
Modelling cosmic ray intensities along the Ulysses trajectory
}

\author{
D. C. Ndiitwani ${ }^{1}$, S. E. S. Ferreira ${ }^{1}$, M. S. Potgieter ${ }^{1}$, and B. Heber ${ }^{2}$ \\ ${ }^{1}$ Unit for Space Physics, School of Physics, North-West University, 2520 Potchefstroom, South Africa \\ ${ }^{2}$ Fachbereich Physik, Universität Osnabrück, Barbarastr. 7, 49069 Osnabrück, Germany
}

Received: 20 October 2004 - Revised: 25 January 2005 - Accepted: 28 January 2005 - Published: 30 March 2005

\begin{abstract}
Time dependent cosmic ray modulation in the inner heliosphere is studied by comparing results from a 2$\mathrm{D}$, time-dependent cosmic ray transport model with Ulysses observations. A compound approach, which combines the effects of the global changes in the heliospheric magnetic field magnitude with drifts to establish a realistic timedependence, in the diffusion and drift coefficients, are used. We show that this model results in realistic cosmic ray modulation from the Ulysses launch (1990) until recently (2004) when compared to 2.5-GV electron and proton and 1.2-GV electron and Helium observations from this spacecraft. This approach is also applied to compute radial gradients present in 2.5-GV cosmic ray electron and protons in the inner heliosphere. The observed latitude dependence for both positive and negative charged particles during both the fast latitude scan periods, corresponding to different solar activity conditions, could also be realistically computed. For this an additional reduction in particle drifts (compared to diffusion) toward solar maximum is needed. This results in a realistic charge-sign dependent modulation at solar maximum and the model is also applied to predict charge-sign dependent modulation up to the next expected solar minimum.
\end{abstract}

Keywords. Interplanetary physics (Cosmic rays; Energetic particles) - Solar physics, Astrophysics and astronomy (Magnetic fields)

\section{Introduction}

The full understanding of solar cycle related changes in the observed cosmic ray intensities over an 11-year cycle is still a major issue in cosmic ray modulation theory. Using a numerical model, it was shown by Perko and Fisk (1983), see also le Roux and Potgieter (1989), that the heliospheric modulation of cosmic rays over long periods requires some form of propagating diffusion barriers. This is especially true when periods of maximum solar modulation are considered when clear step decreases in the intensities were observed.

Correspondence to: S. E. S. Ferreira

(fsksesf@puknet.puk.ac.za)
The largest form of these diffusion barriers is known as global merged interaction regions (GMIRs), as introduced by Burlaga et al. (1993). Equally important to long-term cosmic ray modulation are gradient, curvature and current sheet drifts (Jokipii et al., 1977), as confirmed by comprehensive modeling done by Potgieter et al. (1993) and le Roux and Potgieter (1995). These authors also showed that it was possible to simulate, to the first order, a complete 11-year proton modulation cycle by including a combination of drifts and GMIRs in a comprehensive time-dependent model.

More recently, Cane et al. (1999) and Wibberenz et al. (2002) argued that the cosmic ray step decreases observed at Earth could not be primarily caused by GMIRs because these decreases occurred before any GMIR could form beyond $10 \mathrm{AU}$. Instead, they suggested that time-dependent global changes in the heliospheric magnetic field (HMF) might be responsible for long-term modulation. This approach was modeled and tested by Ferreira (2002), who could simulate an 11-year modulation cycle successfully for cosmic ray observations at neutron monitor energies (e.g. $16 \mathrm{GV}$ ), see also Ferreira and Potgieter (2004). However, for rigidities $<5 \mathrm{GV}$ this approach resulted in far less modulation than what was observed, so that a modified approach was proposed, known as the compound approach. This approach combines the effects of the global changes in the HMF magnitude with drifts, therefore also time dependent current sheet "tilt angles", in order to establish realistic time-dependent diffusion coefficients. The compound model was described by Ferreira (2002); see also Potgieter and Ferreira (2001), Potgieter et al. (2001) and Ferreira and Potgieter (2004). They used this approach to compute realistic global cosmic ray modulation over a full 11-year and 22-year modulation cycle. Although in lesser detail, cosmic ray intensities in the outer heliospheric regions could be reproduced realistically as compared to, for example, Voyager 1 and 2 observations (Ferreira and Potgieter 2004). They concluded that GMIRs could not be ruled out as the cause of the very large step decreases during solar maximum activity in the outer heliosphere. However, they also found that this straightforward approach to global particle drifts resulted in effects that were too large during extreme solar maximum periods when 


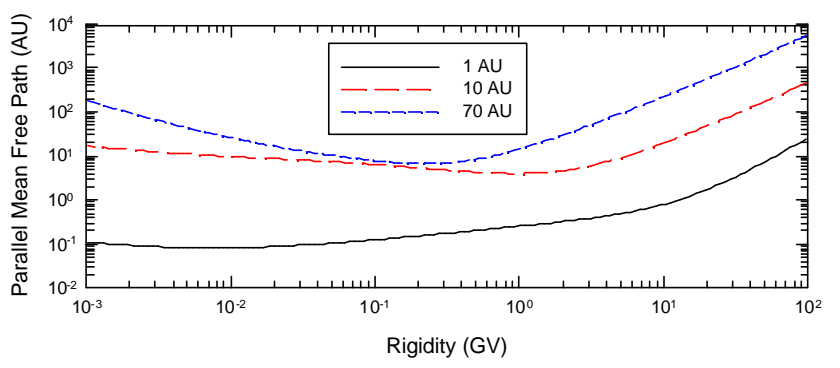

Fig. 1. The parallel mean free path $\left(\lambda_{\|}\right)$as a function of rigidity given by Eq. (2). Shown here are the values at three different radial distances in the equatorial plane.

they considered the finer detail of time-dependent chargesign modulation. In order to reproduce the observed chargesign dependence, they concluded that apart from the timedependence of the diffusion tensor, deduced from the time dependence of the solar magnetic field, the "drift coefficient" needed an additional reduction, compared to diffusion, to even a point of no drifts at solar maximum.

In this work, the focus is on this additional reduction in particle drifts toward solar maximum and what is required in order to simulate more realistic charge-sign dependent modulation as compared to the classic compound modeling done by, for example, Ferreira and Potgieter (2004). In particular, modelling results are compared to $2.5-\mathrm{GV}$ electron and proton intensities and 1.2-GV electron and Helium intensities along the Ulysses trajectory. By computing realistic cosmic ray modulation as compared to Ulysses observations from launch (1990) up to date (2004), the model is further applied to predict charge-sign dependent modulation for the next few years by making reasonable estimates of the modulating parameters. It is shown that the continued analysis of Ulysses observations will be of great importance to fully understand the role of particle drifts, and cosmic ray transport in the $\mathrm{A}<0$ polarity cycle in the next few years. Finally, this modified compound approach is also applied to compute the radial gradients for $2.5-\mathrm{GV}$ cosmic ray electrons and protons in the inner heliosphere.

\section{The transport model and parameters}

To calculate cosmic ray intensities along the Ulysses trajectory, a 2-D time dependent modulation model, as described by le Roux and Potgieter (1989), is used. This model was modified to include the simulation of the heliospheric current sheet as developed by Hattingh and Burger (1995). The model is based on a numerical solution of Parker's (1965) time-dependent transport equation:

$$
\begin{aligned}
\frac{\partial f}{\partial t} & =-\left(\mathbf{V}+\left\langle\mathbf{v}_{D}\right\rangle\right) \cdot \nabla f+\nabla \cdot\left(K_{s} \cdot \nabla f\right) \\
& +\frac{1}{3}(\nabla \cdot \mathbf{V}) \frac{\partial f}{\partial \ln P}+J_{\text {source }},
\end{aligned}
$$

where $f(\mathbf{r}, P, t)$ is the cosmic ray distribution function; $P$ is rigidity, $\mathbf{r}$ is position, $\mathbf{V}$ is the solar wind velocity and $t$ is time. The terms on the right-hand side represent convection, gradient, and curvature drifts, diffusion, adiabatic energy changes, and a source function, respectively. The tensor $\mathbf{K}_{s}$ consists of a parallel diffusion coefficient $\left(K_{\|}\right)$and two perpendicular diffusion coefficients, in the radial direction $\left(K_{\perp r}\right)$ and in the polar direction $\left(K_{\perp \theta}\right)$. The pitch angle averaged guiding center drift velocity for a near isotropic cosmic ray distribution is given by $\left\langle\mathbf{v}_{D}>=\nabla \times\left(K_{A} \mathbf{e}_{B}\right)\right.$, with $\mathbf{e}_{B}=\mathbf{B} / B_{m}$, and $B_{m}$ as the magnitude of the modified background HMF, with $K_{A}$ as the off-diagonal element of the full diffusion tensor.

This equation was solved time-dependently for both the so-called $A>0(\sim 1970-1980 ; \sim 1990-2001)$ and $A<0$ epochs $(\sim 1980-1990 ;>2001)$, using as time-dependent input parameters the time varying "Hoeksema tilt angles", $\alpha$ (see Wilcox Solar Observatory: http://quake.stanford.edu/), and the measured HMF values at Earth (see NSSDC COHOWeb: http://nssdc.gsfc.nasa.gov/cohoweb), as shown in Fig. 2. Note that these parameters which are measured at Earth are propagated outward into the heliosphere with the solar wind speed. Two different Hoeksema-models exist for calculating $\alpha$; the "classic" model uses a line-of-sight boundary condition and the "new" model user a radial boundary condition at the photosphere (Hoeksema, 1992). Ferreira (2002) found that the $\alpha$ with the smallest rate of change over a period of decreasing or increasing solar activity provides the best compatibility with cosmic ray observations see also Ferreira and Potgieter (2003a; 2004). Therefore, in this work, where the modulation of cosmic rays is explored over 22 years, $\alpha$ the values of corresponding to the "new" model are used for periods of increasing solar activity (1976.0-1979.9, 1987.4-1990.0 and 1995.5-2000.0) and $\alpha$ the values of corresponding to the "classic" model are used for periods of decreasing solar activity (1979.9-1987.4 and 1990.0-1995.5).

The outer modulation boundary was assumed at $120 \mathrm{AU}$, where the different local interstellar spectra (LIS) were specified (for electrons the LIS from Langner et al., 2001; for protons the LIS from Moskalenko et al., 2002; for the Helium the LIS from Webber, 1987). The solar wind speed $V$ was assumed to change from $400 \mathrm{~km} \mathrm{~s}^{-1}$ in the equatorial plane (polar angle $\theta=90^{\circ}$ ) to a maximum of $800 \mathrm{~km} \mathrm{~s}^{-1}$ when $\theta=0^{\circ}-60^{\circ}$ and $\theta=120^{\circ}-180^{\circ}$ for solar minimum conditions (McComas et al., 2001), while for solar maximum $V=400 \mathrm{~km} \mathrm{~s}^{-1}$ for all polar angles. The model was assumed to be symmetric with respect to the polar axis. The effects of time-dependence in the latitude dependence of the solar wind speed on the modulation of cosmic rays, in particular for low energy electrons, were illustrated by Ferreira et al. (2003b).

For the parallel diffusion coefficient we assume:

$K_{\|}=\frac{v}{3} \lambda_{\|}(r, P)$ 
Here

$\lambda_{\|}(r, P)=\lambda_{1}(r, P) \times \lambda_{2}(r, P) \times f_{2}(t)$ with $f_{2}(t)=\left(\frac{B_{0}}{B(t)}\right)^{n}$,

where

$\lambda_{1}(r, P)=\frac{5}{3}\left[\begin{array}{l}(0.0106 \mathrm{AU}) \times\left(\left(P / P_{0}\right)^{1 / 3}+\frac{3.57}{\left(0.511^{2}+\left(P / P_{0}\right)^{2}\right)^{1 / 4}}\right) \\ +\left(r / r_{0}\right)^{1.4} \times 10^{-9}\left(P / P_{0}\right)^{2}\end{array}\right]$

and

$\lambda_{2}(r, P)=\frac{c_{1}(P)+0.08}{c_{1}(P) \times\left(r / r_{0}\right)^{-2.30}+0.08 \times\left(r / r_{0}\right)^{0.37}}$

with

$c_{1}(P)=83.0\left(\frac{0.02}{1000 P / P_{0}}\right)^{0.75}$

In Eq. (2) $r_{0}=1 \mathrm{AU}, P_{0}=1 \mathrm{MV}$ and $\lambda_{1}$ (in $\mathrm{AU}$ ) give the rigidity (in MV) dependence of $\lambda_{\|}$at Earth, as given by Teufel and Schlickeiser (2002) for the damping model of dynamical turbulence (see also Bieber et al., 1994).

These theoretical studies usually concentrate on the region between the Sun and the Earth, so that the radial dependence beyond $1 \mathrm{AU}$ of the diffusion coefficients is not calculated. In this work we assume the radial dependence which was calculated by, for example, Ferreira (2002) and Ferreira et al. (2004) and given by $\lambda_{2}$, which gave realistic electron modulation at all energies of interest. The term at the end of $\lambda_{1}$ was inserted to assist us in constructing the radial dependence of $\lambda_{\|}$in the rest of the heliosphere and has no effect at $1 \mathrm{AU}$. The parallel mean free path given by Eq. (2) is shown in Fig. 1 as a function of rigidity for three different radial distances in the heliosphere. For distances beyond Earth, $\lambda_{\|}$increases significantly up to $\sim 10 \mathrm{AU}$, and gradually thereafter. This is because different models for turbulence dominates in different parts of the heliosphere. (For details concerning these models, see Zank et al. 1998). The function $f_{2}(t)$, in Eq. (2) (Ferreira and Potgieter 2004), determines the timedependence of all the diffusion coefficients and is discussed below.

Concerning perpendicular diffusion (see, e.g. Stawicki 2004; le Roux et al., 1999) it has become standard practice when using numerical modulation models to scale $K_{\perp}$ as $K_{\|}$(e.g. Kóta and Jokipii, 1998; Burger et al., 2000). For a theoretical motivation, see le Roux et al. (1999). For $K_{\perp r}$ and $K_{\perp \theta}$ we assumed that

$K_{\perp r}=0.02\left(\frac{P}{P_{0}}\right)^{0.3} K_{\|}$and $K_{\perp \theta} / K_{\|}=b F(\theta)$

with $b=0.02$. These expressions results in $K_{\perp r} / K_{\|} \geq 0.02$ for $P \geq 1 \mathrm{GV}$ and $K_{\perp \theta} / K_{\|}=0.02$ (in the equatorial plane), as required by the simulations done by Giacalone and Jokipii (1999). Furthermore, Burger et al. (2000) illustrated that in order to produce the correct magnitude and rigidity dependence of the observed latitudinal cosmic ray proton density gradient by Ulysses, enhanced latitudinal transport

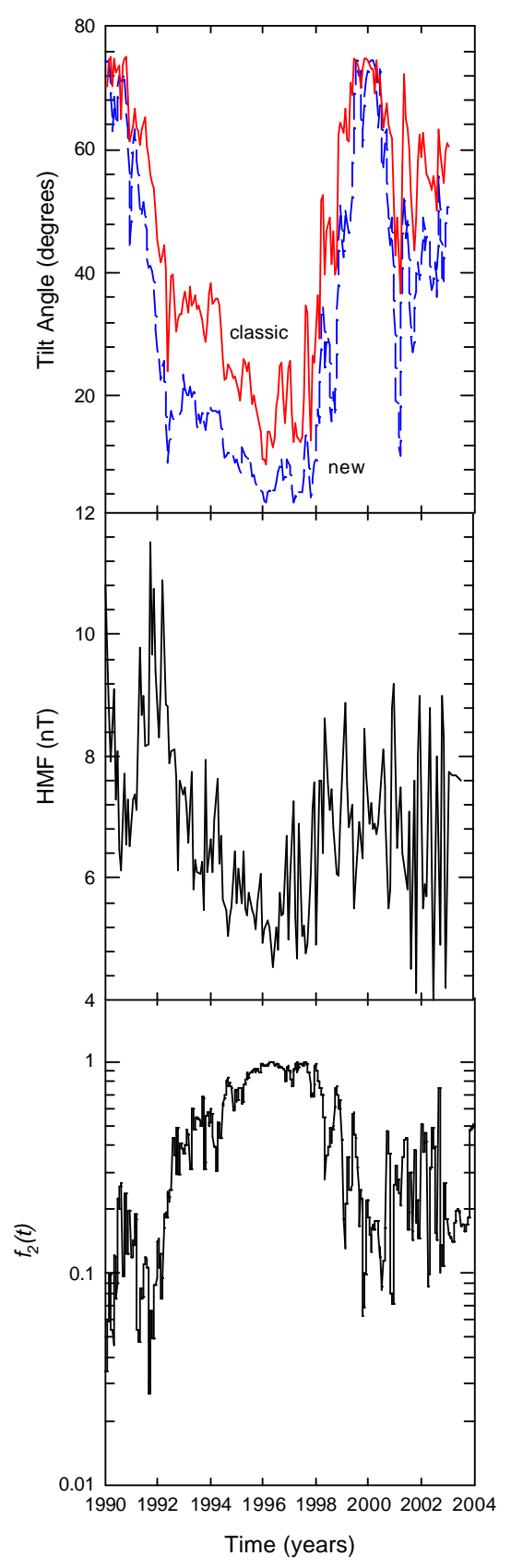

Fig. 2. Top Panel: The time varying "Hoeksema tilt angles", $\alpha$ (see Wilcox Solar Observatory: http://quake.stanford.edu/). Middle Panel: The measured HMF values at Earth (see NSSDC COHOWeb: http://nssdc.gsfc.nasa.gov/cohoweb. Bottom Panel: The corresponding time dependence $f_{2}(t)$ of the diffusion coefficients, see Eq. (2), for the period 1990 to 2004.

is required (see also Potgieter et al., 1997). This is accomplished by increasing $K_{\perp \theta}$ toward the poles by a factor 6 , via the function $\mathrm{F}(\theta)$, with respect to the value in the equatorial plane. For details, see, e.g. Burger et al. (2000), Ferreira et al. (2001). 

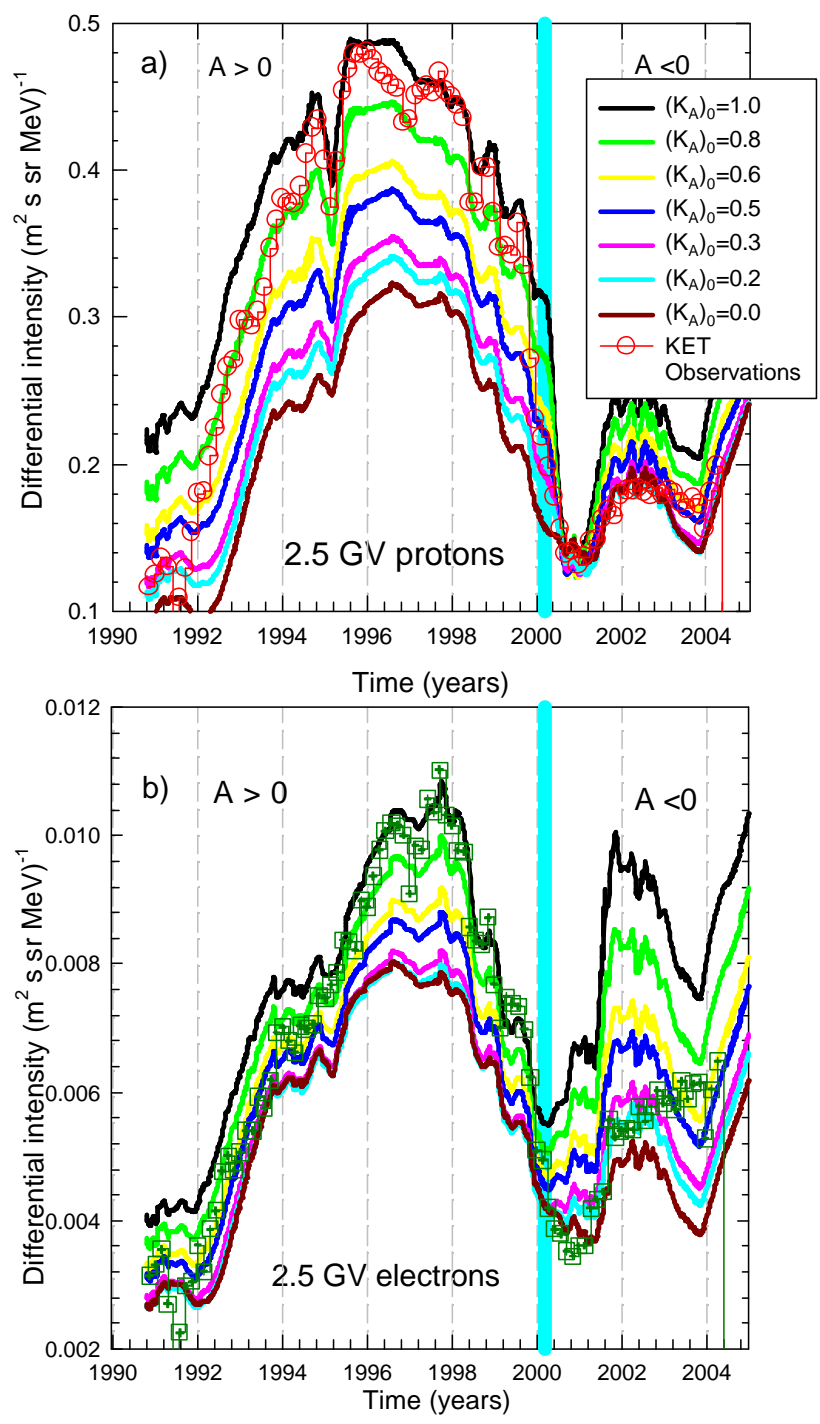

Fig. 3. Solutions of the compound modeling for different values of the drift constant $\left(K_{A}\right)_{0}$ in Eq. (4). Shown from top to bottom are model results corresponding to $\left(K_{A}\right)_{0}=1.0,0.8,0.6,0.5$, $0.3,0.2,0.0$, respectively. Shown in Fig. 3 a are different computed 2.5-GV proton intensities and in Fig. 3b 2.5-GV electron intensities along the Ulysses trajectory in comparison with Ulysses/KET observations (Heber et al., 2003), with protons in the energy range from $250-2000 \mathrm{MeV}$ and electrons from $400-4000 \mathrm{MeV}$. For all model calculations the $\mathrm{HMF}$ switches polarity (from $\mathrm{A}>0$ to $\mathrm{A}<0$ ) at 2000.2 , as indicated by the thick vertical line.

The drift coefficient is changed time-dependently as follows:

$$
\begin{aligned}
K_{A} & =\left(K_{A}\right)_{0} \frac{K_{\text {drift }}(P)}{3 B_{m}} f_{2}(t) \text { with } K_{\text {drift }}(P) \\
& =\beta P \frac{D_{f a k} P^{2}}{D_{\text {fak }} P^{2}+1}
\end{aligned}
$$

and with $B_{m}$ as the Parker HMF but modified in the heliospheric polar regions similar to what Jokipii and Kóta (1989) proposed; $D_{f a k}=10.0$ in units of (rigidity) ${ }^{-2}$ which causes drifts to be somewhat reduced at lower rigidities as explained by Burger et al. (2000); $\left(K_{A}\right)_{0}=1.0$, and $\beta$ is the ratio between the particle speed to the speed of light.

The function $f_{2}(t)$, in Eq. (2), with $B_{0}=5 \mathrm{nT}$, determines the time-dependence of all the diffusion coefficients as a function of the measured HMF at Earth, $B(t)$, which is interpreted to be an elementary representation of turbulence over a solar cycle. Ferreira and Potgieter (2004) illustrated that $n$ could not be a constant and had to change with time (and rigidity), where this time dependence must be related to solar activity. From a drift point of view, the obvious choice was the time-varying $\alpha$. The compound approach for longterm cosmic ray modulation was consequently proposed by assuming $\mathrm{n}=\alpha / \alpha_{0}$, with $\alpha_{0}$ as a constant which may vary with rigidity. In Fig. 2, this time dependence in the diffusion coefficients $f_{2}(t)$ (Eq. (2) with $\alpha_{0}=18$ ) is shown for the period 1990 up to 2000. Evidently, the diffusion coefficients are larger ( $\sim$ a factor of 10 or more depending on the rigidity) at solar minimum than at solar maximum - see also Cummings and Stone (2001) - and are highly time-dependent. These time dependent changes are propagated outwards into the heliosphere at the solar wind speed causing time-dependent diffusive barriers to move from $1 \mathrm{AU}$ and beyond. Note that these barriers may also merge, eventually, but no merging was allowed for this work. Also, the spatially 2-D nature of the model means that we average the cosmic ray intensities over one solar rotation. Therefore, the effects of recurrent features of the solar wind speed, like corotating interaction regions, on the modulation of high energy cosmic rays are not considered (see Potgieter et al., 1993; Potgieter and le Roux, 1994 and Kissmann et al., 2003). However, these merged interaction regions contribute little to long-term modulation (e.g. Potgieter and le Roux, 1994) and the concept of global merged interaction regions (propagating diffusive barriers) is needed to simulate cosmic ray modulation successfully.

\section{Results and discussion}

To compute a realistic amplitude in the 11-year and 22-year modulation cycles of cosmic ray intensities, Ferreira (2002) and Ferreira and Potgieter (2004) assumed a highly timedependent $f_{2}(t)$ in Eq. (2) where $\mathrm{n}=\alpha / \alpha_{0}$ and $\alpha_{0}=11$. Although resulting in a realistic computed modulation amplitude over a solar cycle, these authors showed that there was still too much drift present for intermediate to large solar activity when charge-sign dependent modulation are studied. Therefore, in this work, the first computed results focus on the level of drifts required to reproduce proton and electron observations from the Ulysses/KET instrument (Heber et al., 2003) from 1991 to 2004. In Fig. 3 the results from the compound model are shown for different values of the drift constant $\left(K_{A}\right)_{0}$ in Eq. (4). Shown from top to bottom are solutions with $\left(K_{A}\right)_{0}=1.0,0.8,0.6,0.5,0.3,0.2$, 0.0 , respectively. In comparison protons in the energy range from $250-2000 \mathrm{MeV}$ and electrons from $400-4000 \mathrm{MeV}$ are 
shown. In Fig. 3a the corresponding computed 2.5-GV proton intensities are shown and in Fig. $3 b$ the corresponding 2.5-GV electron intensities along the Ulysses trajectory in comparison with the observations. For all the computations it is assumed that the HMF switches polarity (from $\mathrm{A}>0$ to $\mathrm{A}<0)$ at 2000.2 , as indicated by the thick vertical line. In contrast to Ferreira and Potgieter (2004) a smaller amplitude in the time dependence, $f_{2}(t)$, in the diffusion (and drift) parameters is assumed by setting $\alpha_{0}=18$ in $\mathrm{n}=\alpha / \alpha_{0}$ in Eq. (2). Comparing the solutions with diminishing drifts to the observations in Fig. 3 demonstrates that using $\left(K_{A}\right)_{0}=1.0$ (the top line) results in realistic modulation for solar minimum conditions (1996-1998) but for intermediate to maximum activity the computations are much higher than observed. In order to improve this, $\left(K_{A}\right)_{0}$ has to be reduced progressively toward solar maximum, even to the level of zero drifts at extreme maximum activity. This results in realistic levels of modulation over the 11-year cycle for both the $2.5-\mathrm{GV}$ protons and electrons. This means that the "drift coefficient" is reduced significantly more with time than the other diffusion coefficients, in order to compute realistic charge-sign dependent modulation up to when the HMF polarity reverses. Therefore, $f_{2}(t)$, as shown in Fig. 2, combined with the reduction of $\left(K_{A}\right)_{0}$, is indicative of the level of drifts as a function of time over a full 11-year modulation cycle.

Figure 4 shows the total amount of drifts needed in the model (red line) given by $f_{2}(t)\left(K_{A}\right)_{0}$, to compute a modulation compatible to Ulysses/KET observations for the various stages of the solar cycle. The percentage drifts (on the lefthand side) is shown with respect to the varied tilt angle $\alpha$ as used in the model (see discussion above), for a proxy for solar activity. Shown here is that the percentage of drifts correlates with solar activity, with large drifts needed for solar minimum, and almost no drifts for solar maximum. (Note that values are shown from small at the top, to large at the bottom, on the right-hand side of the figure). During solar minimum periods, drifts are obviously large, varying between $80 \%$ and $100 \%$ for at least three years around solar minimum. As soon as intermediate solar activity starts with a decrease in tilt angle, drifts follow suit to increase the modulation. During solar maximum conditions drifts reduced to less than $10 \%$ for most of this period.

Figure 5 shows computed intensities along the Ulysses trajectory using the model with the modified compound approach and parameters as described above. In comparison Ulysses KET observations (Heber et al., 2003) are shown for 2.5-GV protons and electrons, and for 1.2-GV He and electrons (See also Clem et al., 2002) in Figs. 3a-d, respectively. The HMF switches polarity (from $\mathrm{A}>0$ to $\mathrm{A}<0$ ) at 2000.2, as indicated by the thick line. The vertical dashed lines indicate the beginning and the end of the first fast latitude scan (FLS1) and the second fast latitude scan (FLS2) of the Ulysses spacecraft. These results illustrate that the model gives realistic modulation for the different cosmic ray species at the given rigidities. During the FLS1 (close before solar minimum), the 2.5-GV proton and 1.2-GV Helium intensities show a noticeable latitudinal dependence, and which coincided with

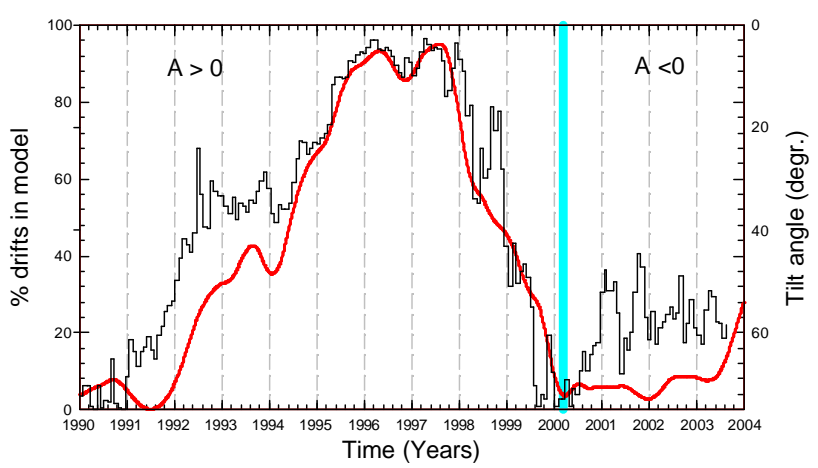

Fig. 4. The percentage of drifts (red line) in the model, described by $f_{2}(t)\left(K_{A}\right)_{0}$, that gives realistic modulation for various stages of the solar cycle for both the 2.5-GV electron and protons, as shown in Fig. 3. As a proxy for solar activity the tilt angles as used in the model are shown by the black line. Note the scale on the right-hand side.

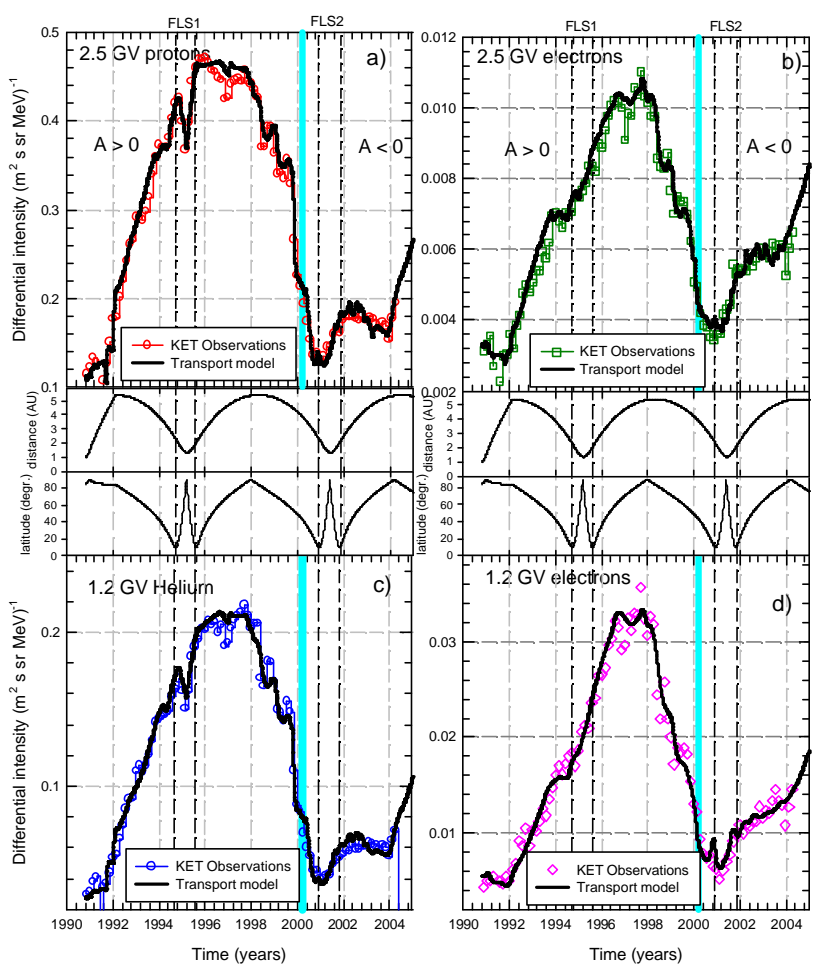

Fig. 5. The computed intensities along the Ulysses trajectory with model parameters described in the text. In comparison Ulysses KET observations (Heber et al. 2003) are shown for Fig. 3a (2.5-GV protons), Fig. 3b (2.5-GV electrons), Fig. 3c (1.2 GV He) and Fig. 3d (1.2-GV electrons) (see also Clem et al., 2002), respectively. For all model calculations it is assumed that the HMF switches polarity (from $\mathrm{A}>0$ to $\mathrm{A}<0$ ) at 2000.2 , as indicated by the thick vertical line. Shown as vertical dashed lines are the beginning and the end of the first fast latitude scan (FLS1) and the second fast latitude scan (FLS2) of the Ulysses spacecraft, of which the trajectory in radial distance and latitude is shown in the middle panels. 


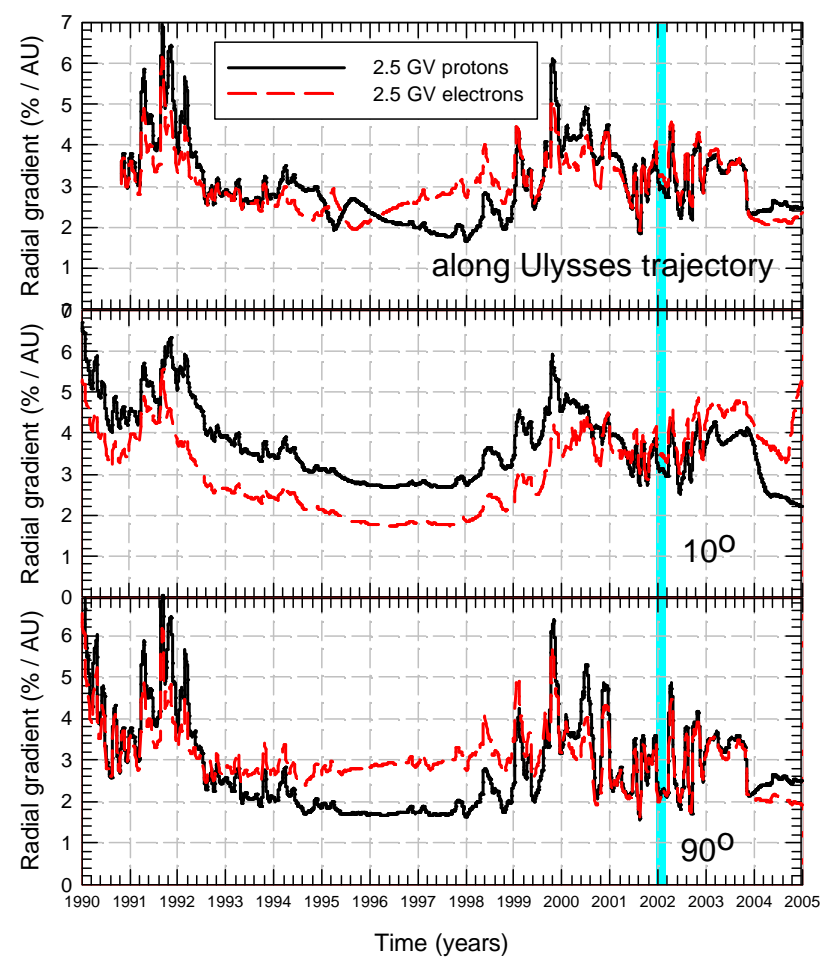

Fig. 6. Computed radial gradients as a function of time in the inner heliosphere based on results shown in Fig. 5. Values are shown along the Ulysses trajectory (top panel), near the heliospheric poles (middle panel) and in the equatorial plane (bottom panel), for 2.5-GV electrons (red dashed line) and 2.5-GV protons (black solid line). For all model calculations it is assumed that the $\mathrm{HMF}$ switches polarity (from $\mathrm{A}>0$ to $\mathrm{A}<0$ ) at 2000.2.

solar maximum activity disappears for the FLS2 period. The time dependence in the latitudinal dependence is modeled realistically by assuming $K_{\perp \theta}$, as in Eq. (3). The 1.2-GV and 2.5-GV electron observations show no latitude dependence for the FSL1 and FLS2, a modulation feature which is also successfully reproduced by the compound approach. Also shown in Fig. 5 is that the modulation steps occurring in the Ulysses/KET observations, especially in 1994 and between 1998 and 2000, can be reproduced with the model.

Also modelled realistically are the recovery times after solar maximum, which started around 2001 in the observed cosmic ray intensities and clearly differ depending on the charge of the cosmic rays. For both the 1.2- and 2.5-GV electrons the observed and computed intensities increase significantly after/during solar maximum conditions, compared to the 1.2-GV Helium and proton intensities. These particle exhibit longer recovery times. This is due to drift effects, since a positively charged particles transport into the inner heliosphere is expected to be faster over the poles, as in the $\mathrm{A}>0$, than along the HCS, as in the $\mathrm{A}<0$.

Apart from computing realistic latitudinal gradients, as shown indirectly in Fig. 5, it is shown in Fig. 6 that this model can also compute radial gradients of which the features are comparable to observations. To compute this, the procedure as described in Heber et al. (2002) is followed. Concerning the observations, Clem et al. (2002) reported that the radial gradient of cosmic ray electrons in the heliosphere at rigidities of 1.2 and $2.5 \mathrm{GV}$ from 1 to $5 \mathrm{AU}$ appears to be the same as those for positive particles of the same rigidity. Furthermore, Heber et al., 2002 showed that the radial gradient of 2.5-GV protons increased from 2.2\%/AU to 3.5\%/AU from solar minimum to maximum, respectively, making a time-dependent determination of the electron radial gradients mandatory. It was also shown by, for example, Fujii and McDonald (2001) that the radial gradient for protons varies with polarity; it is larger in an $\mathrm{A}<0$ than in an $\mathrm{A}>0$ solar magnetic epoch

Figure 6 shows the time-dependent radial gradients in the inner heliosphere, computed with the compund approach. It is based on the computed modulation of protons and electrons as shown in the previous figure. Values are shown along the Ulysses trajectory, (top panel), near the heliospheric poles (middle panel) and in the equatorial plane (bottom panel) for electrons (red dashed line) and protons (black solid line). Shown here is that in the equatorial regions, and for solar minimum conditions (1995-1998), the electrons have a radial gradient of $\sim 2.9 \% / \mathrm{AU}$ while the protons have $\sim 1.9 \% / \mathrm{AU}$, for the $\mathrm{A}>0$ polarity cycle. The latter is in good agreement with values reported by Heber et al. (2002). For solar maximum there is a general increase, apart from a high variablity, in the radial gradients for both particle species in the equatorial regions. The values are also quite similar for protons and electrons, primarily caused by the small levels of drifts present. For these extreme solar activity periods both the $2.5-\mathrm{GV}$ electrons and protons have a very fluctuating gradient, varying between $3-4 \% / \mathrm{AU}$ (see also Heber et al., 2002), up to even 5\%/AU on shorter time scales. Similar values were found by Heber et al. (1993) for the solar maximum periods of 1990-1992, where the radial gradient was found to be $4.9 \pm 1.3 \% / \mathrm{AU}$. Interesting is that after 2000.2, when the HMF switches polarity from $A>0$ to $\mathrm{A}<0$ in the model, a general decrease in the radial gradients occur, and the electron radial gradient becomes larger than the proton gradient, indicative of how the radial gradients vary with HMF polarity.

For the polar regions, shown in Fig. 6, almost the opposite occurs. Here the electrons have a smaller gradient of $\sim 1.9 \%$ /AU compared to protons at $\sim 2.9 \%$ /AU for the solar minimum conditions in the $\mathrm{A}>0$ polarity cycle. For solar maximum, the gradients of both particle species increase, as do the differences between them. However, there values differ between 4-5\%/AU for both the electrons and the protons. After the polarity reversal in 2000.2 the electron radial gradient becomes larger than the proton gradient.

Concerning charge-sign dependent modulation and the HMF polarity reversal Fig. 7 shows the computed 1.2$\mathrm{GV}$ e/He and 2.5-GV e/p ratios along the Ulysses trajectory. In comparison the Ulysses/KET observations (Heber et al., 2003) are shown. Values are normalized to 1.0 at the start of the Ulysses mission. It follows that the decrease in the observed and computed e/p and e/He toward solar minimum 


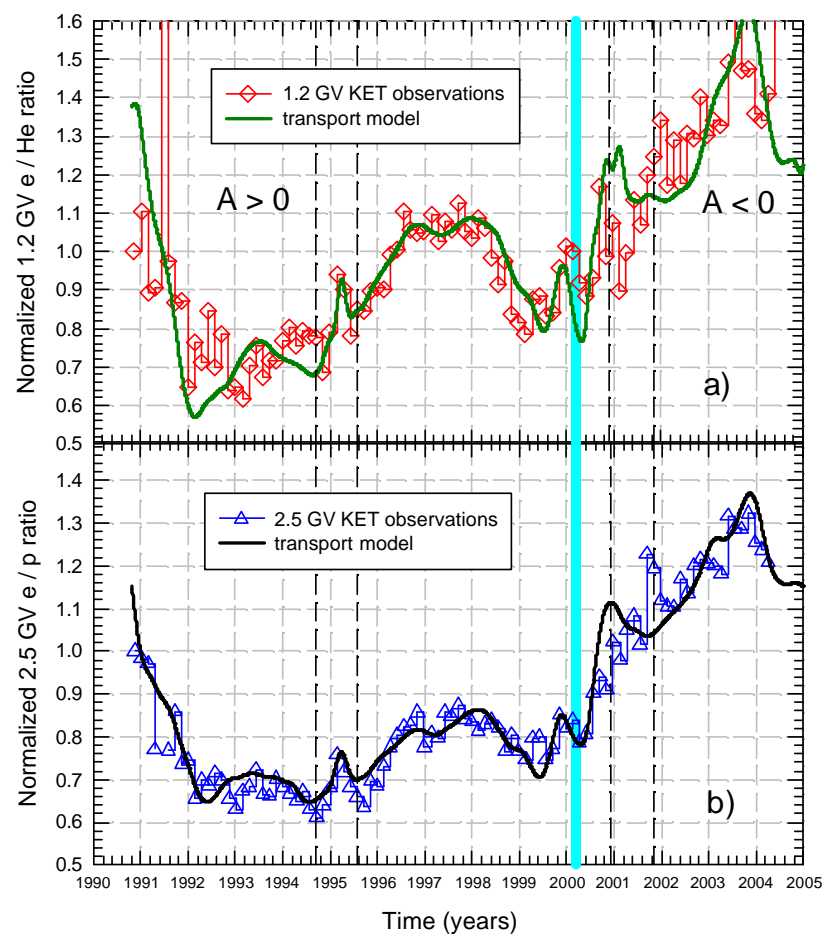

Fig. 7. Computed 1.2-GV e/He and $2.5-\mathrm{GV}$ e/p ratios along the Ulysses trajectory. In comparison the Ulysses/KET observations (Heber et al., 2003) are shown. The HMF switches polarity, from $\mathrm{A}>0$ to $\mathrm{A}<0$, in the model at 2000.2, as indicated by the thick vertical line. As before, the vertical dashed lines indicate the beginning and the end of first (FLS1) and second fast latitude scan (FLS2) of the Ulysses spacecraft. Values are normalized to 1.0 at the start of the Ulysses mission in 1991.

for the A>0 polarity cycle from 1990 to 1995 can be attributed to charge-sign dependent modulation from drift effects, resulting in the peak-(electrons) and plateau (protons) like shapes of cosmic ray intensities over the 11-year modulation cycle. In both panels the latitudinal dependence of the positively charged particles for solar minimum periods is also visible. The latitudinal dependence of these particles along the Ulysses trajectory results in an "additional" decrease of the e/p and the e/He toward solar minimum compared to, for example, measurements at Earth for the first fast latitude scan (FLS1) period. When the HMF polarity reverses after 2000 there is a steady increase in the computed intensities up to 2004, whereafter a sudden decrease is expected (see discussions below). The value of scaling down drift effects in the model via the modified compound approach presented here can be seen when these computations are compared to other authors, e.g. Burger and Potgieter (1999), Ferreira (2002), etc. In contrast to the computed steady increase in these ratios toward solar minimum after a polarity reversal, these traditional models predict large, sudden increases or decreases which are not observed.

Figure 8 shows computed and observed 2.5-GV e/p (not normalized) along the Ulysses trajectory. Three model solutions are shown corresponding to three different specified

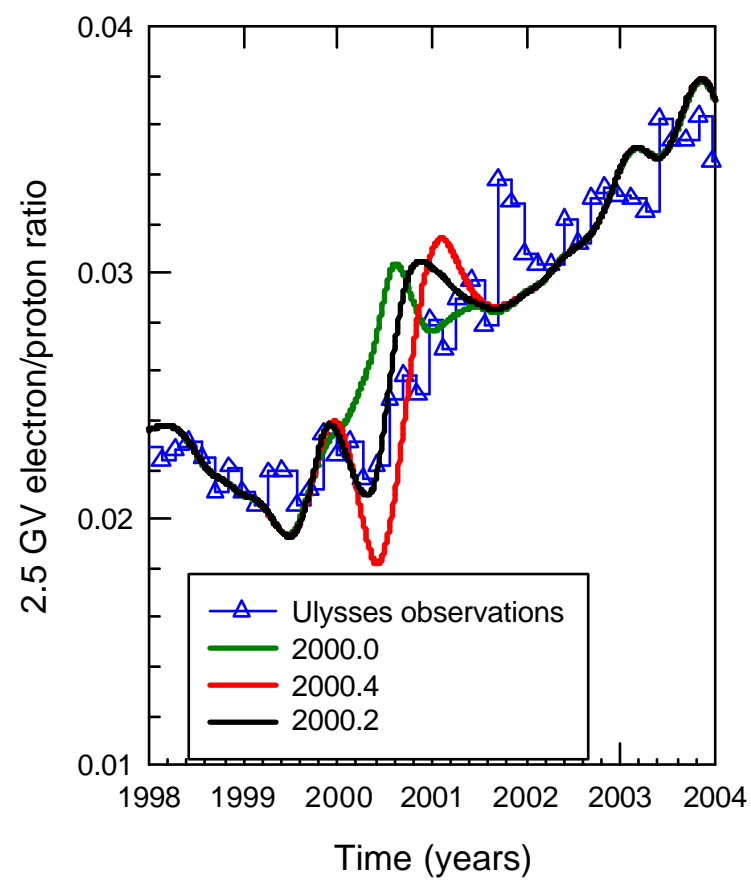

Fig. 8. Computed and observed 2.5-GV e/p (not normalized) along the Ulysses trajectory. Three model solutions are shown corresponding to different scenarios of fixed polarity reversal times in the model. The black line corresponds to a HMF polarity reversal in 2000.2, as in Fig. 6, the red line to 2000.4 and the green line to 2000.0 .

times of polarity reversal, from $\mathrm{A}>0$ to $\mathrm{A}<0$. The black line corresponds to 2000.2, as in Fig. 6, the red line to 2000.4 and the green line to 2000.0. Shown here is that the magnetic field polarity reversal influence model computations, although only for approximately 1 year. This is the time scale needed for information to travel from the inner heliosphere to the heliopause. After 2000.4, these solutions converge again because the whole heliosphere is then filled with heliospheric magnetic fields corresponding to the $\mathrm{A}<0$ polarity cycle. Evidently, the choice of 2000.2 from a cosmic ray modulation perspective is optimal. For earlier values the computed $\mathrm{e} / \mathrm{p}$ increases too fast, and for latter values the computed e/p decreases too much after 2000, which is not observed. However, in these modulation models this polarity reversal occurs at a specified time-step, on the order of a solar rotation, which may be contrasted to observations which predict a longer time period of several months (see, e.g. Jones et al., 2003)

Finally, the modified compound approach is used to predict the $2.5-\mathrm{GV}$ e/p along the Ulysses trajectory up to the next solar minimum expected at $\sim 2007$. For this it is assumed that the tilt angle will decrease gradually from present values to $\sim 20^{\circ}$ in 2007. This is shown in Fig. 9. Values are shown from 1998 up to 2007. The dark line separates the present values from the predicted values. Also shown in the lower left panel is the heliospheric magnetic field magnitude, and the predicted values. Here we assumed that the HMF 

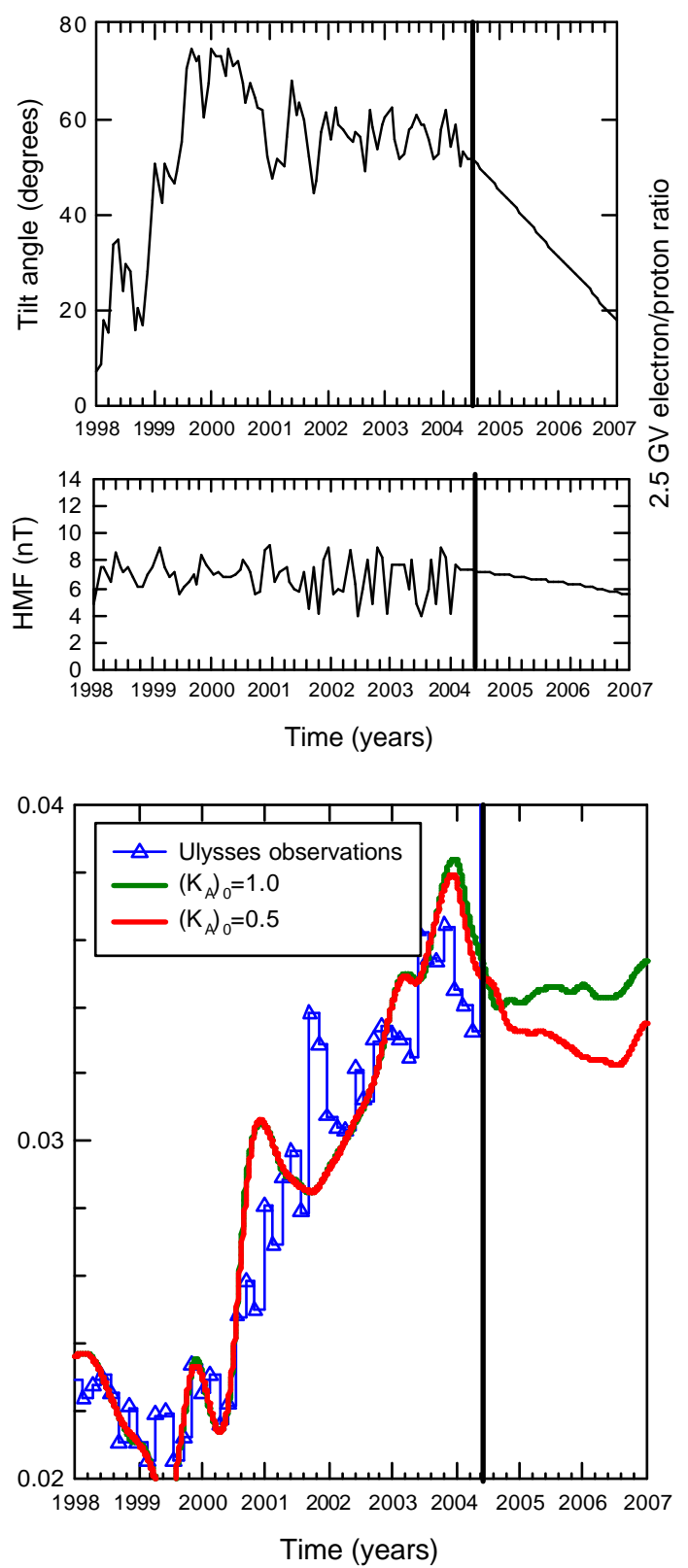

Fig. 9. Computed 2.5-GV e/p along the Ulysses trajectory including predictions up to the next solar minimum, assumed at $\sim 2007$. It is assumed that the tilt angle will decrease gradually as shown in the top panel. In the middle panel is the HMF magnitude, and the predicted values. The dark line separates the observed values from the predicted values. Shown in the bottom panel is the computed 2.5-GV e/p (not normalized) along the Ulysses trajectory with most recent observations. Two different computations are shown corresponding to two different $\left(K_{A}\right)_{0}$ assumed after 2004.5, in Eq. (4).

magnitude will stay relatively constant. Smoothed values are chosen for the predictions because we found that at these energies, the cosmic ray modulation is not that sensitive to the finer details of the short-scale variations in tilt angle and HMF magnitude. These aspects are currently under investigation. Shown in Fig. 9 is the computed 2.5-GV e/p (not normalized) along the Ulysses trajectory with most recent observations. Two different computations, corresponding to two different assumed $\left(K_{A}\right)_{0}$ after 2004.5 in Eq. (4), are shown. Figure 9 illustrates how drifts recover from almost no drifts at solar maximum to full drifts at solar minimum. Already in $2004,\left(K_{A}\right)_{0}=0.5$ compute realistic modulation, corresponding to $\sim 40 \%$ drifts $\left(f_{2}(t)\left(K_{A}\right)_{0}\right)$, as shown in Fig. 4. Also shown as an upper value is $\left(K_{A}\right)_{0}=1.0$, where no additional decrease in the drift coefficient, compared to the diffusion coefficients, was assumed. It follows that the $\left(K_{A}\right)_{0}=0.5$ scenario (red line) will result in a gradual decrease in the computed e/p up to 2006.5, where afterwards an increase occurs. For the scenario corresponding to $\left(K_{A}\right)_{0}=1.0$ (green line) the predicted $\mathrm{e} / \mathrm{p}$ will stay mostly constant for the next few years. Future data from Ulysses observations, for example, the e/p, will play an important role in determining the amount of drifts present in cosmic ray modulation and in understanding particle transport in the $\mathrm{A}<0$ polarity cycle.

\section{Summary and conclusions}

In this work, a time dependent numerical model, based on the Parker (1965) transport equation, was utilized to calculate realistic cosmic ray intensities along the Ulysses trajectory from launch (1990) up to (2004) and to compare to Ulysses observations. This approach is similar to previous efforts by e.g. Ferreira (2002), Potgieter and Ferreira (2001), Potgieter et al. (2001) and Ferreira and Potgieter (2004), who utilized a compound approach, which combines the effects of the global changes in the HMF magnitude with drifts, therefore also time dependent current sheet "tilt angles", to establish a realistic time-dependent diffusion and drift coefficients. These results were then used as input parameters to a time-dependent, 2-D cosmic ray transport model. However, it was shown by the above-mentioned authors that this approach could largely compute realistic global cosmic ray modulation over a full 11- and 22-year modulation cycle, but still results in drift effects toward solar maximum which are too large when charge-sign dependent modulation is considered.

In this work, we have shown that an additional reduction in particle drifts (compared to diffusion) toward solar maximum is needed to produce, for example, a more realistic chargesign dependent modulation. Shown in Fig. 4 was the total percentage of drifts as needed in the transport model to compute realistic modulation. Shown here is that this correlates with solar activity, with large drifts needed for solar minimum, and almost no drifts for solar maximum. For example, during solar minimum periods drifts are large, varying between $80 \%$ and $100 \%$ for at least three years around solar minimum. As soon as intermediate solar activity starts, with the decrease in tilt angle, the percentage of drifts follows suit to increase the modulation. During solar maximum conditions, drifts reduced to less than $10 \%$ for most of the period, to compute a realistic modulation along the Ulysses trajectory, when compared with observations (2.5-GV electron and 
proton and 1.2-GV electron and Helium) along the Ulysses trajectory, as was shown in Fig. 5.

Concerning the computed latitudinal gradients it was shown that during the first fast latitude scan (FLS1) of the Ulysses spacecraft (which coincides with solar minimum), the 2.5-GV proton and 1.2-GV Helium intensities show an observable latitudinal dependence, which disappears for the second latitude scan (FLS2) period (which coincides with solar maximum). This was also modeled realistically. In contrast, both the 1.2-GV and 2.5-GV electron observations shows no latitude dependence for both FSL1 and FLS2, a feature which was also successfully reproduced by the compound approach. From these results radial gradients in the inner heliosphere could be computed and shown in Fig. 6. It was shown that in the equatorial regions, and for solar minimum conditions, the $2.5-\mathrm{GV}$ electrons have a radial gradient of $\sim 2.9 \% / \mathrm{AU}$ while for the protons it is $\sim 1.9 \% / \mathrm{AU}$ for the $\mathrm{A}>0$ polarity cycle. The latter is in good agreement with values reported by Heber et al. (2002). For solar maximum there is a general increase in the radial gradient for both particle species in the equatorial regions due to the small amount of drifts present. For these extreme solar activity periods both the 2.5-GV electrons and protons have a large fluctuating gradient varying between 3-4\%/AU (see also Heber et al., 1993; Heber et al., 2002), increasing to even 5\%/AU for shorter time scales. After 2000.2 when the HMF polarity changes in the model, from $\mathrm{A}>0$ to $\mathrm{A}<0$, there is a general decrease in the computed radial gradient, with the radial gradient of electrons becoming larger than that for protons, indicating that the gradients vary with HMF polarity.

Lastly, charge-sign dependent modulation of cosmic rays along the Ulysses trajectory, and the effect of the HMF polarity reversal were also considered. Figure 7 showed a steady decrease in the observed and computed 2.5-GV e/p and 1.2$\mathrm{GVe} / \mathrm{He}$ ratios toward solar minimum and the steady increase thereafter, for the $\mathrm{A}>0$ polarity cycle. This is in contrast to traditional models, for example, Burger and Potgieter (1999), Ferreira (2002), which predict large, sudden increases or decreases, which are not observed. This indicates that drifts need to be scaled down to compute realistic modulation. Also shown in Fig. 8 was that from a cosmic ray perspective, the polarity reversal from $A>0$ to $A<0$ should be around 2000.2. Finally, the modified compound approach was also used to predict the $2.5-\mathrm{GV}$ e/p along the Ulysses trajectory up to the next solar minimum expected at $\sim 2007$. We showed in Fig. 9 that this ratio will gradually decrease from present values up to 2006.5, where afterward an increase is predicted. Future data from Ulysses observations, for example, the e/p, will play an important role in determining the amount of drifts present in cosmic ray modulation and in understanding particle transport in the $\mathrm{A}<0$ polarity cycle.

Acknowledgements. The authors wish to thank H. Fichtner and G. Wibberenz for valuable discussions. This material is based upon work partially supported by the National Research Foundation under grant number 2053475.

Topical Editor R. Forsyth thanks I. Usoskin and B. Ball for their help in evaluating this paper.

\section{References}

Burger, R. A. and Potgieter, M. S.: The effect of large current sheet tilt angles in numerical modulation models: a theoretical assessment, Proc. 26th International Cosmic Ray Conference, 7, 1316, 1999.

Burger, R. A., Potgieter, M. S., and Heber, B.: Rigidity dependence of cosmic-ray proton latitudinal gradients measured by the Ulysses spacecraft: Implications for the diffusion tensor, J. Geophys. Res., 105, 27 447-27 456, 2000.

Bieber, J. W., Matthaeus, W. H., Smith, C. W., Wanner, W., Kallenrode, M. B., and Wibberenz, G.: Proton and electron mean free paths: The Palmer consensus revisited, J. Astrophys., 420, $294-$ 306, 1994.

Burlaga, L. F., McDonald, F. B., and Ness, N. F.: Cosmic ray modulation and the distant heliospheric magnetic field: Voyager 1 and 2 observations from 1986 to 1989, J. Geophys. Res., 98, 1-11, 1993.

Cane, H. V., Wibberenz, G., Richardson, I. G., and von Rosenvinge, T. T.: Cosmic ray modulation and the solar magnetic field, Geophys. Res. Lett., 26, 565-568, 1999.

Clem, J. M., Evenson, P., and Heber, B.: Cosmic electron gradients in the inner heliosphere, Geophys. Res. Lett., 29, 2096-2100, 2002.

Cummings, A. C. and Stone, E. C.: Onset of solar modulation in the outer heliosphere as seen in anomalous cosmic rays, Proc. 27th International Cosmic Ray Conference, 10, 4251-4254, 2001.

Ferreira, S. E. S.: The heliospheric transport of galactic cosmic rays and jovian electrons, Ph.D. thesis, Potchefstroom University, South Africa, 2002.

Ferreira, S. E. S. and Potgieter, M .S.: Long term cosmic ray modulation in the heliosphere, J. Astrophys., 603, 744-752, 2004.

Ferreira, S. E. S. and Potgieter, M. S.: On the tilt angles of the heliospheric current sheet used in modulation models over a 22year cosmic ray modulation cycle, Adv. Space Res., 32(4), 657662, 2003a.

Ferreira, S. E. S., Potgieter, M. S., and Moeketsi, D.: Modulation effects of a changing solar wind speed profile on low-energy electrons, Adv. Space Res., 32(4), 675-680, 2003b.

Ferreira, S. E. S., Potgieter, M. S., Burger, R. A., Heber, B., and Fichtner, H.: Modulation of jovian and galactic electrons in the heliosphere 1. Latitudinal transport of a few-MeV electrons, J. Geophys. Res., 106, 24 979-24 988, 2001.

Fujii, Z. and McDonald, F. B.: Radial diffusion coefficients and the distance to the modulation boundary for galactic and anomalous cosmic rays, Adv. Space Res., 27(3), 559-564, 2001.

Giacalone, J. and Jokipii, J. R.: The transport of cosmic rays across a turbulent magnetic field, J. Astrophys., 520, 204-214, 1999.

Hattingh, M. and Burger, R. A.: A new simulated wavy neutral sheet drift model , Adv. Space Res., 13(9), 213-216, 1995.

Heber, B., Raviart, A., Paizis, C., Bailk, M., Droge, W., Ducros, R. et al.: Modulation of galactic cosmic ray particles observed onboard the Ulysses spacecraft, Proc. 23rd. International Cosmic Ray Conference, 461-464, 1993.

Heber, B., Wibberenz, G., Potgieter, M. S., and Burger, R. A.: Ulysses Cosmic Ray and Solar Particle Investigation/Kiel Electron Telescope observations: Charge-sign dependence and spatial gradients during the 1990-2000 A >0 polarity magnetic cycle, J. Geophys. Res., 107, SSH2-1, 2002.

Heber, B., Clem, J. M., Muller-Mellin, R., Kunow, H., Ferreira, S. E. S., and Potgieter, M. S.: Evolution of the galactic cosmic ray electron to proton ratio: Ulysses COSPIN/KET observations, 
Geophys. Res. Lett., 30, ULY 6-1-4, 2003.

Hoeksema, J. T.: Large scale structure of the heliospheric magnetic field; 1976-1991, in: Solar Wind Seven, edited by: Marsch, E. and Schwenn, R., Pergamon, Oxford, p191-196, 1992.

Jokipii, J. R, Levy, E. H., and Hubbard, W. B.: Effects of particle drift on cosmic ray transport, I General properties and application to solar modulation, J. Astrophys., 213, 861-868, 1977.

Jokipii, J. R. and Kóta, J.: The polar heliospheric magnetic field, Geophys. Res. Lett., 16, 1-4, 1989.

Jones, G. H., Balogh, A., and Smith, E. J.: Solar magnetic field reversal as seen at Ulysses, Geophys. Res. Lett., 30, ULY2-1-4, 2003.

Kissmann, R., Fichtner, H., Heber, B., Ferreira, S. E. S., and Potgieter, M. S.: First results of a new 3-D model of the timedependent modulation of elctrons in the heliosphere, Adv. Space. Res., 32(4), 681-686, 2003.

Kóta, J. and Jokipii, J. R.: Modelling of 3-D corotating cosmic ray structures in the heliosphere, Space Sci. Rev., 83, 137-145, 1998.

Langner, U. W., De Jager, O. C., and Potgieter, M. S.: On the local interstellar spectrum for cosmic ray electrons, Adv. Space Res., 27(3), 517-520, 2001.

le Roux, J. A. and Potgieter, M. S.: A numerical model for a cosmicray modulation barrier in the outer heliosphere. Astron. Astrophys., 209, 406-410, 1989.

le Roux, J. A. and Potgieter, M. S.: The simulation of complete 11 and 22 year modulation cycles for CRs in the heliosphere using a drift model with global merged interaction regions, J. Astrophys., 442, 847-851, 1995.

le Roux, J. A., Zank, G. P., and Ptuskin, V. S.: An evaluation of perpendicular diffusion models regarding cosmic ray modulation on the basis of a hydromagnetic description for solar wind turbulence, J. Geophys. Res., 104, 24 845-24 962, 1999.

McComas, D. J., Goldstein, R., Gosling, J. T., and Skoug, R. M.: Ulysses' second orbit: remarkably different solar wind, Space Sci. Rev., 97, 99-103, 2001.

Moskalenko, I. V., Strong, A. W., Ormes, J. F., and Potgieter, M. S.: Secondary antiprotons and propagation of cosmic rays in the galaxy and heliosphere, J. Astrophys., 565, 280-296, 2002.
Parker, E. N.: The passage of energetic charged particles through interplanetary space, Planet. Space Sci., 13, 9-49, 1965.

Perko, J. S. and Fisk, L. A.: Solar modulation of galactic cosmic rays. 5. Time dependent modulation, J. Geophys. Res., 88, 90339036, 1983.

Potgieter, M. S. and Ferreira, S. E. S.: Modulation of cosmic rays in the heliosphere over 11 and 22 year cycles: A modeling perspective, Adv. Space Res., 27(3), 481-492, 2001.

Potgieter, M. S. and le Roux, J. A.: The long-term heliospheric modulation of galactic cosmic rays according to a timedependent drift model with merged interaction regions, J. Astrophys., 423, 817-827, 1994.

Potgieter, M. S., Burger, R. A., and Ferreira, S. E. S.: Modulation of cosmic rays in the heliosphere from solar minimum to maximum: a theoretical perspective, Space Sci. Rev., 97, 295-307, 2001.

Potgieter, M. S., Haasbroek, L. J., Heber, B., and Ferrando, P.: The modelling of the latitude dependence of cosmic ray protons and electrons in the inner heliosphere, Adv. Space Res., 19(6), 901920, 1997.

Potgieter, M. S., le Roux, J. A., Burlaga, L. F., and McDonald, F. B.: The role of merged interaction regions and drifts in the heliospheric modulation of cosmic rays beyond $20 \mathrm{AU}$ : A computer simulation, J. Astrophys., 403, 760-768, 1993.

Stawicki, O.: On Quasilinear Perpendicular Diffusion, Astron. Astrophys., in press, 2005.

Teufel, A. and Schlickeiser, R.: Analytic calculation of the parallel mean free path of heliospheric cosmic rays, I. Dynamical magnetic slab turbulence and random sweeping slab turbulence, Astron. Astrophys, 393, 703-715, 2002.

Webber, W. R.: Cosmic ray proton and helium spectra from 5200 GV measured with a magnetic spectrometer, Proc. 21th. Inter. Cosmic Ray Conf., 1, 325-328, 1987.

Wibberenz, G., Richardson, I. G., and Cane, H. V.: A simple concept for modeling cosmic ray modulation in the inner heliosphere during solar cycles 20-23, J. Geophys. Res., 107, SSH 5-1, 2002.

Zank, G. P., Matthaeus, W. H., Bieber, J. W., and Moraal, H.: The radial and latitudinal dependence of the cosmic ray diffusion tensor in the heliosphere, J. Geophys. Res., 103, 2085-2098, 1998. 